\title{
Security in Emergency Departments: Spatial Analysis of the Physical Environment
}

\author{
Erica Del hagen \\ Department of Interior Design, College of Design Construction and Planning
}

Faculty Mentor: Shabboo Valipoor, Department of Interior Design

\begin{abstract}
Emergency departments (EDs) are one of the most complex clinical areas in the healthcare system and one of the most dangerous workplaces. Evidence shows that healthcare professionals who work in EDs are more prone to violence from patients and visitors than those working in other healthcare settings. While many studies have highlighted organizational and operational policies to minimize the risk of violence against healthcare workers, very few studies have focused on the role of facility design. This research aimed to (1) gain an understanding of environmental design features in EDs that may impact the occurrence of violet events, and (2) propose potential environmental design scenarios for future examination of ED design through primary research. Peer-reviewed and gray literature on ED design were reviewed, and case examples of ED facility design projects were identified from online sources and were spatially analyzed. Findings from the literature suggested that entry zones of EDs, including entrance doors and vestibules for walk-in patients, reception areas/desks, and waiting areas are associated with the most security issues. Based on this finding, the spatial analysis was limited to the entry zones. The design examples were categorized based on their spatial patterns and visibility properties. This typology of entry layouts helped with developing three design scenarios with different levels of visibility. The design scenarios represent the most common layouts of entry design in ED facilities. A walk-through video of each scenario is developed for future research. A future study can identify the perceived security level of each scenario by presenting them to healthcare workers and security professionals working in EDs. This research highlights the importance of interior environmental design in improving security and reducing the risk of violent behavior against healthcare workers in EDs.
\end{abstract}

Keywords: Emergency departments, interior environment, spatial design, security, visibility

\section{Introduction}

Emergency departments (EDs) are the most complex clinical settings in the modern hospital infrastructure. Several factors contribute to this complexity, including the large variety of patients visiting, the wide variety of services provided, and provision of continuous, 24-hours-aday seven days a week (24/7) care. In recent years, the opioid crisis, reduction in the number of EDs nationwide, and a shortage in ED medical staff have made the ED situation even more complex and contributed to overcrowding in EDs (Gillespie et al., 2017). Overcrowding and long 
waiting times lead to patient dissatisfaction and, in many cases, to violent behavior. Healthcare professionals in EDs are prone to violence from patients and visitors. According to a study by Lyneham (2001), each of the 266 ED nurses who participanted in a descriptive exploratory survey reported experiencing some form of either verbal or physical violence at least weekly.

\section{The Structure of Emergency Care}

EDs offer a wide range of services as they adapt and change to new challenges on a daily basis. EDs may provide five different levels of care. Level I trauma centers provide the highest level of care. These centers are equipped with the largest range of specialties of care. Level II centers are equipped to handle most surgical cases and illnesses; this department is also open for 24-hour care. Level III EDs are able to treat quick emergencies or ones that are not lifethreatening. Finally, Levels IV and V mainly provide initial evaluation, stabilization and prepare the patients to be transported to a larger hospital if needed. Lower-level EDs, Levels IV-V, can be free-standing from the main hospital. This allows for less crowding, as well as a greater reach into neighborhoods with less access to the main hospital.

Using the Emergency Severity Index (ESI), emergency departments determine the priority of incoming patients into three main categories, non-urgent, urgent and emergency patients. It is critical for staff to do a rapid assessment of patients in order to determine which patient(s) are at highest risk and to facilitate patient placement. Patients entering the emergency department arrive via three basic modes, ambulance, walk-in, or public service. Ambulance patient can arrive in vehicles by ground or by air, walk-in patients arrive by foot or private vehicle, and public service patients can arrive through the assistance of a police car or other service vehicles. Patients arriving by Emergency Medical Services (EMS) need to be evaluated quickly and be transferred to a patient room. Steps of care upon arrival to the emergency department are classified into five stages: triage, registration, treatment, reevaluation, and discharge or admission or transmission to the hospital.

When first arriving to the emergency department, triage occurs whereby staff assess the patient's ESI level to identify who should proceed first for treatment. Next, registration occurs whereby staff will proceed to collect information about the patient as well as consent to receive treatment during their visit. This stage is commonly performed at the registration desk, before moving the patient to the ED patient room; however, it may also be conducted at the patient's bedside if the patient was transferred for treatment immediately upon arrival. Once a patient is 
moved to the designated treatment space, the care team will assess the patient's condition and needs and will start the best course of treatment. After treatment, and re-evaluation in some cases, it will be determined if the patient should be discharged or admitted to the hospital for further treatment. Most EDs staff a ratio of one nurse per three patients, whereas a doctor may cover anywhere from eight patients at a time up to twelve depending on the department (Huddy, 2016).

\section{Design Configurations}

According to Zilm (2007), the three most commonly used layout designs for EDs are called: ballroom, matrix, and the perimeter corridor. The most traditional type is the ballroom (Figure 1). The intention of this model is to allow for the nurses to have views into the patient rooms from a centralized work station, identified as "charting/support" in the model. The key flaw of this model is the limited number of visible patients at any given time. On average, a ballroom model only allows for around 17 patient rooms to be placed around a centralized work station. With this model, there is an inability to adjust and accommodate to an influx of patients unless the model is replicated, which makes it hard to manage staffing in response to opening new pods (each organizational model). The matrix model (Figure 2) allows for more flexibility. This model is more adaptable to patient fluctuations and allows for nurses to move freely and add support where necessary in accordance with any given situation. In this model, multiple entry portals can be provided, and nurses can group patients by the required type of care. In addition, in response to larger volume of patients, lateral expansion and sharing staff would be possible. Finally, a layout option highly favored by ED staff, is the linear model, also known as a perimeter corridor

model (Figure 3). This model allows for nursing staff to flow freely between the patient rooms in a linear motion while maintaining a continuous visual access to the patients. In addition to funneling the general walk-in patients to a single side of the emergency department, this plan segregates the patients arriving via ambulance to allocate them to the adjacent side of the department. 


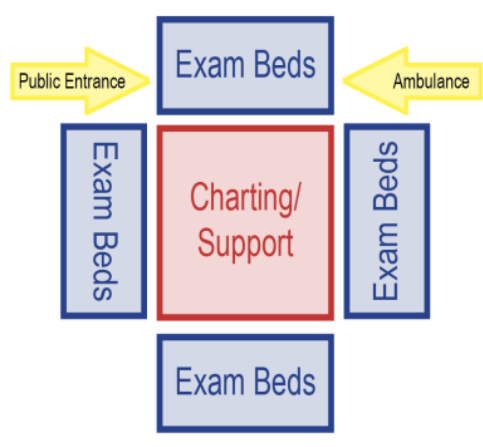

Figure 1. The Traditional "Ballroom" Organizational Model

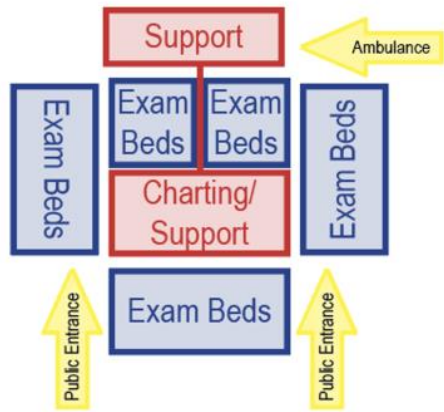

Figure 2. The "Matrix" Organizational Model

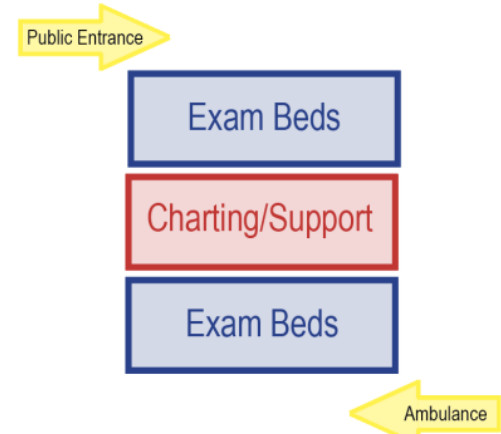

Figure 3. The "Linear" or "Perimeter Corridor Organizational Model

\section{Violence in Eds}

Studies have shown that EDs are the most frequent locations for violence against healthcare workers (Behnam et al., 2011). Many factors contribute to this, including overcrowding, long wait times, substance abuse, high level of stress, and 24-hour service (Zilm, 2007). Repeated violent events at the workplace increase the level of stress among workers and lead to job dissatisfaction, lower quality of care, and in many cases, burnout (Rozo \& Olson, 2017). In a study by Gates et al. (2011 ), 94\% of ED nurses suffered at least one Post-traumatic Stress Disorder (PTSD) symptom. While many studies have highlighted organizational and operational policies to minimize the risk of violence against healthcare workers, very few studies have focused on the role of facility design. The aim of this study was to (1) gain a deeper understanding of environmental design features in EDs that may impact the occurrence of violet events, and (2) propose potential environmental design scenarios for future examination of ED design through primary research.

\section{Methods}

Peer-reviewed and gray literature on ED design were reviewed, and case examples of ED facility design projects were spatially analyzed. A Google search was performed using to the keywords such as "emergency department design," "layout," "floor plan," "organizational model," "space planning," "entry," and "lobby" to identify drawings, images of ED entry zones, renderings, floor plans, and other relevant images. The search was further screened to eliminate images that did not clearly show the relationship between the different spaces. In the next step, the case examples identified through image searching were categorized based on their spatial properties and possible vulnerability to violent events. This typology of layouts along with 
findings from the literature helped with developing three design scenarios for future examination. The design scenarios were visualized using Autodesk Revit 2021. A walk-through video of each scenario was also developed.

\section{Review of Literature}

Findings from the literature suggested that in EDs, the following environment-related conditions may contribute to aggressive behavior by patients/visitors: constant surveillance of patients by cameras (Lau et al., 2012), lack of privacy (AbuAlRub \& Al Khawaldeh, 2014), lack of enough patient beds as well as delivering care in crowded corridors (Angland et al., 2014), lack of visual access from registration and waiting areas to the clinical zone entry (Pati et al., 2016). Previous studies also found that entry zones of EDs, including entrance doors and vestibules for walk-in patients, reception areas/desks, and waiting areas are associated with the most security issues resulting from aggressive behavior towards ED staff (Gharaveis et al., 2017; Lau et al., 2012; Pane et al., 1991; Pati et al., 2016; Shaw, 2015). Pati et al. (2016) highlighted the importance of visibility from the entrance to the security station, triage, and reception desk. They found that visibility of the security station enhances the perception of safety and security.

The following design strategies that are suggested to reduce the risk of violent events in EDs can be implemented in the entry zone: reception desks with deep countertops and sloped sides to prevent violent patients/visitors from reaching over easily and harming the staff (Huddy, 2016); shatter proof glass barriers with openings to prevent a person from attempting to go over the counter or throw objects (Shaw, 2015), positive distractions (e.g., television, artwork, music) to reduce stress level (Pane et al., 1991), screens to provide up-to-date information and reduce frustration (Pane et al., 1991), comfortable and flexible furniture options to alleviate physical strain of sitting for an extended period of time in long waiting time cases (Pane et al., 1991), comfortable temperatures, pleasurable smells, and access to natural light (Eggert et al., 2014). McPhaul et al. (2008) identified the importance of visibility from control points in EDs, and Bowers et al. (2009) recommended a minimum number of vantage points.

In addition to ED entry zone issues, relevant facility design topics can be drawn from other areas of literature. Providing staff with clear vantage points of the ED entry (walk-in and ambulatory entrances), triage, registration, and waiting lobby, enables them to monitor situations as they arise quickly. In addition, placing the security station near the entrance, in view of these 
zones, allows for access at any time and leads to a perception of safety among staff . This means that they would feel more at safe while working (Pati et al., 2016). It is recommended that doors between different spaces should remain locked at all times, such as the doors separating the waiting room and the treatment bay. They must be accessible only to staff with key access. In addition to being locked, these doors should be automatically closing, set to close in 15 seconds (Shaw, 2015). A known safe vestibule design is the Man Trap. This passageway is set between two doors, in which the second door will not be able to be opened until the first door is to fully close (Huddy, 2016). Both of these considerations are used to prevent people from following staff into the department. Staff badges have a variety of different safety uses. Badges can be used to gain entry to restricted areas, each of these badges should also be equipped with Real Time Location Systems (RTLS). RTLS works similarly to radio frequency, allowing the staff member's locations to be tracked while in the hospital (Huddy, 2016). This technology, paired with panic buttons next to the badge, allows for security to immediately be alerted of the location in the hospital where assistance is needed. In the case that a dangerous situation arises, it is important to have multiple exit points in a room to avoid being trapped. Designing work stations that are 6 to 8 feet long with breaks between, allows for staff to walk between desks and reduces the risk of staff getting trapped in case of an emergency (Huddy, 2016).

Suggestions for other ED areas include designing triage rooms with two doors (Fay et al., 2017) and establishing clustering day and night areas to facilitate monitoring (McPhaul et al., 2008). In comparison to smaller work pods, staff prefer to work in larger pods (Pati et al., 2016). These large pods allow for them to have visual access to their peers. It also enhances communication and increases the sense of security within staff members, minimizing isolation. Having the staff pod located in a centralized location within the intake zones allows for an increase in patient observation across the department floor. Designing semi-open work stations, allows for the patients and visitors to connect to the staff, while still meeting the needs for visual transparency throughout the department. To meet the needs of visual and auditory transparency, different types of barriers are put to use. Privacy screens between registration seats, varying array of transparent wall barriers, and doors that have windows.

Hospital staff have expressed their concern over the threat of weapons and dangerous objects being brought into the department and used against them by agitated patients and visitors. While metal detectors are favorable and suggested, there are several considerations to be addressed 
(Huddy, 2016). Due to the time it takes for a single person to pass through the metal detector, it can cause a line to form, preventing walk-in patients from being quickly triaged. To bypass the consequent complications, adding an additional metal detector can alleviate the pressure of the line without a second security guard needing to be present. However, the equipment required for machines of these kind take up a lot of space, therefore putting more than one in a preexisting space may not be an option. The staffing expenses are the primary concern when implementing these entry precautions. Locating staff near the security station will reduce processing times. This would also be helpful in case of a medical emergency while patients are waiting in long lines. Patients arriving by EMS will also need to be screened by security. The most efficient way to do so is using hand-held metal detectors. A private search room is also beneficial (Huddy, 2016).

\section{Results}

Based on the review of literature, the spatial analysis was limited to the entry zone. The entry zone as described in these case studies includes the relation between entrance door, reception desk, and waiting area. The internet search of case examples included photos of EDs, renderings of design projects, and floor plans. The search resulted in 22 images after excluding those with unclear entry zone designs. The remaining images were grouped into six categories based on the layout of their entry zones. Next, a relationship diagram was created for each category to allow for identifying similar patterns and merging similar layouts together (see Figure 4). The result was three main entry zone layout types with different relationships between entrance, reception desk, waiting area, security station, and triage rooms (see Figure 5). The three layouts represent the most implemented similarities found in the ED designs found in Figure 4.
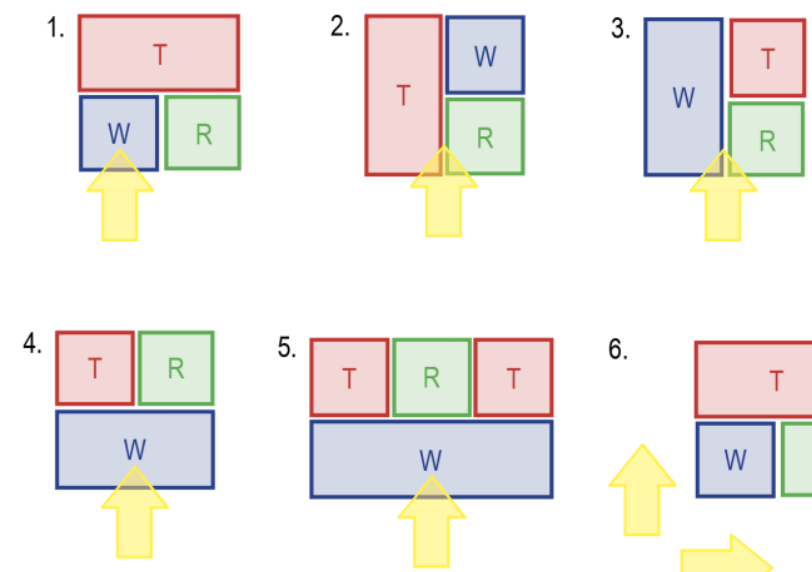

6.

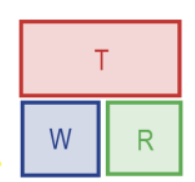

Figure 4. Common zoning in ED design 

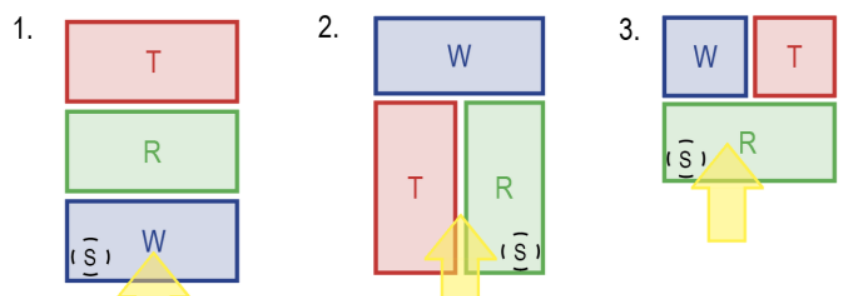

Figure 5. Merged diagrams of zoning in ED design

\section{Design Scenarios}

Three design scenarios were created as walk-through videos to enable examining the space from different vantage points and from the eyes of receptionists, security guards, clinicians, and walk-in patients. Figures 6-8 illustrate still renderings of the three scenarios. The QR codes of videos can be used for future examination of ED designs.
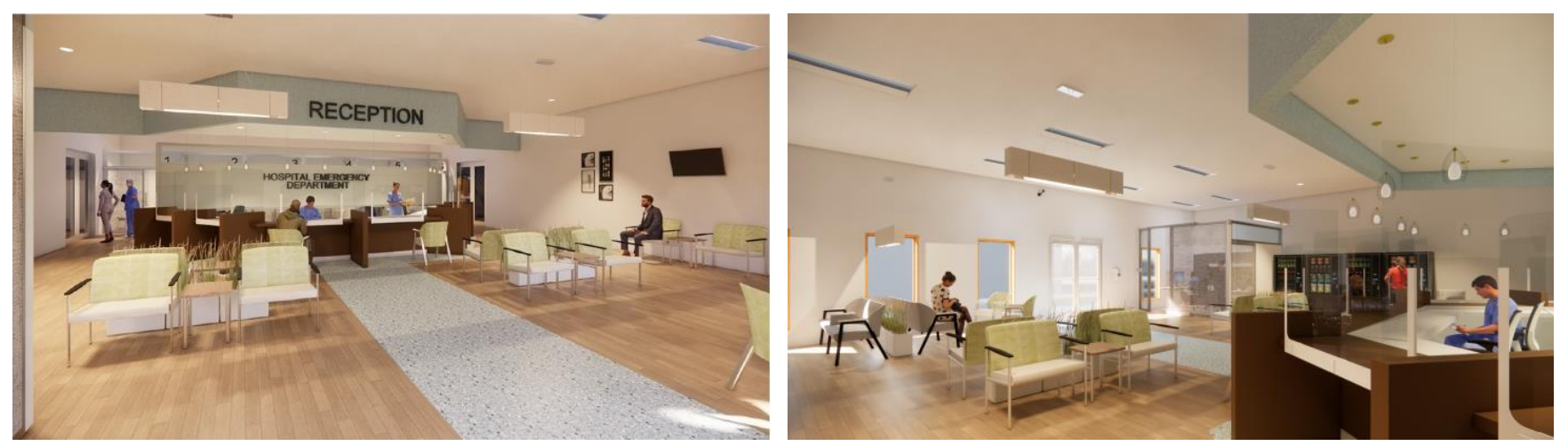

Figure 6. Design Scenario 1 opens to the waiting area located on either side of the centralized reception, with the triage rooms located behind the reception desk.
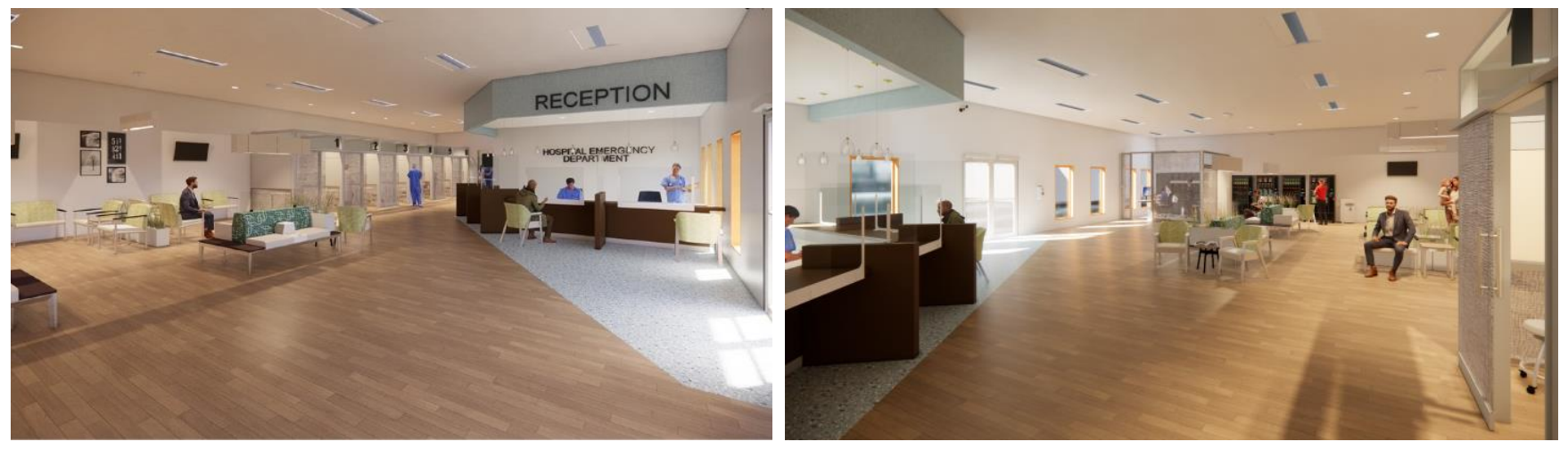

Figure 7. Design Scenario 2, opens to the reception on the right, and the waiting area on the left, while the triage rooms span the right back wall. 

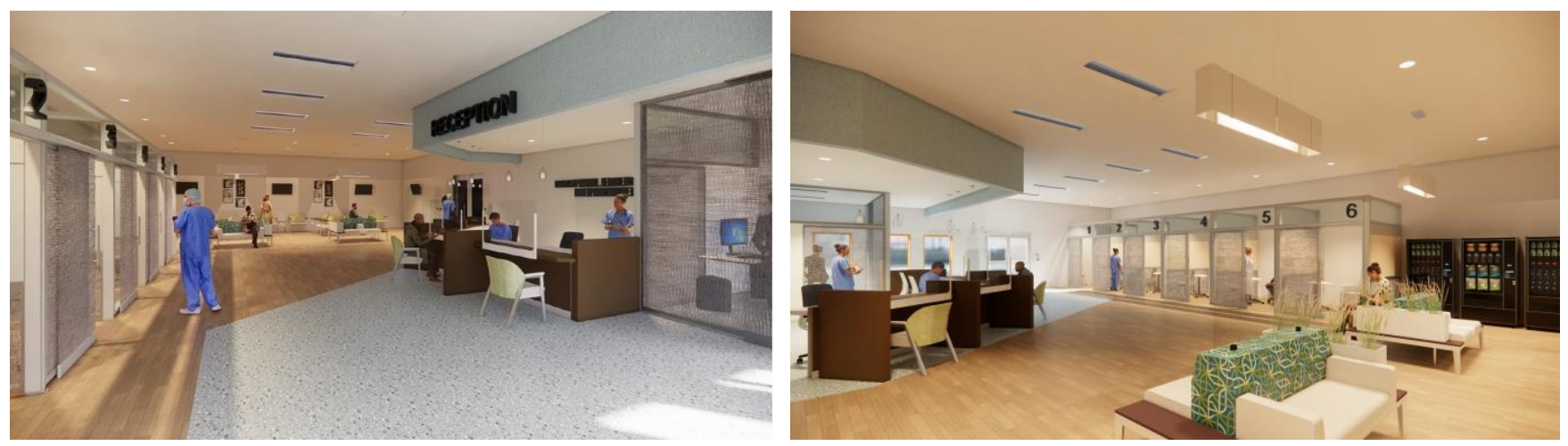

Figure 8. Design Scenario 3, the security booth followed by the reception desk are located to the right, and the triage rooms are located on the adjacent wall. The waiting area is located directly beyond the reception and triage.

\section{Conclusion}

This research highlights the importance of interior environmental design in improving security and reducing the risk of violent behavior against healthcare workers in EDs. The scenarios developed in this study provide an opportunity to further examine the spatial properties of the most vulnerable areas in the most implemented designs of EDs. In a future study, the design scenarios will be presented to healthcare workers and security professionals working in EDs and their perceived security level will be evaluated.

\section{References}

AbuAlRub, R. F., \& Al Khawaldeh, A. T. (2014). Workplace physical violence among hospital nurses and physicians in underserved areas in Jordan. Journal of Clinical Nursing, 23(13-14), 19371947.

Angland, S., Dowling, M., \& Casey, D. (2014). Nurses' perceptions of the factors which cause violence and aggression in the emergency department: A qualitative study. International Emergency Nursing, 22(3), 134-139.

Behnam, M., Tillotson, R. D., Davis, S. M., \& Hobbs, G. R. (2011). Violence in the emergency department: A national survey of emergency medicine residents and attending physicians. The Journal of Emergency Medicine, 40(5), 565-579. https://doi.org/10.1016/j.jemermed.2009.11.007

Bowers, L., Allan, T., Simpson, A., Jones, J., Van Der Merwe, M., \& Jeffery, D. (2009). Identifying key factors associated with aggression on acute inpatient psychiatric wards. Issues in Mental Health Nursing, 30(4), 260-271. https://doi.org/10.1080/01612840802710829 
Eggert, J. E., Kelly, S. P., Margiotta, D. T., Hegvik, D. K., Vaher, K. A., \& Kaya, R. T. (2014). Personenvironment interaction in a new secure forensic state psychiatric hospital. Behavioral Sciences \& the Law, 32(4), 527-538.

Fay, L., Carll-White, A., \& Harrell, J. (2017). Coming full cycle: Linking POE findings to design application. Health Environments Research \& Design Journal, 10(3), 83-98. https://doi.org/10.1177/1937586716672856

Gates, D. M., Gillespie, G. L., \& Succop, P. (2011). Violence against nurses and its impact on stress and productivity. Nurs Econ, 29(2), 59-66.

Gharaveis, A., Hamilton, D. K., Pati, D., \& Shepley, M. (2017). The impact of visibility on teamwork, collaborative communication, and security in emergency departments: An exploratory study. Health Environments Research \& Design Journal. https://doi.org/10.1177/1937586717735290

Gillespie, G. L., Pekar, B., Byczkowski, T. L., \& Fisher, B. S. (2017). Worker, workplace, and community/environmental risk factors for workplace violence in emergency departments. Archives of environmental \& occupational health, 72(2), 79-86.

Huddy, J. (2016). Emergency Department Design: A Practical Guide To Planning For The Future, 2nd Ed. (2nd ed.).

Lau, J. B. C., Magarey, J., \& Wiechula, R. (2012). Violence in the emergency department: a ethnographic study (part I). International emergency nursing, 20(2), 69-75.

Lyneham, J. (2001). Violence in New South Wales emergency departments. Australian Journal of Advanced Nursing, 18(2), 8-20.

McPhaul, K. M., London, M., Murrett, K., Flannery, K., Rosen, J., \& Lipscomb, J. (2008). Environmental evaluation for workplace violence in healthcare and social services. Journal of Safety Research, 39(2), 237-250. https://doi.org/10.1016/j.jsr.

Pane, G. A., Winiarski, A. M., \& Salness, K. A. (1991). Aggression directed toward emergency department staff at a university teaching hospital. Annals of Emergency Medicine, 20(3), 283286.

Pati, D., Pati, S., \& Harvey, T. E., Jr. (2016). Security implications of physical design attributes in the emergency department. Health Environments Research \& Design Journal, 9(4), 50-63.

Rozo, J. A., Olson, D. M., Thu, H., \& Stutzman, S. E. (2017). Situational factors associated with burnout among emergency department nurses. Workplace health \& safety, 65(6), 262-265.

Shaw, J. (2015). Staff perceptions of workplace violence in a pediatric emergency department. Work, 51(1), 39-49.

Zilm, F. (2007). A New Era of Emergency Care Planning and Design. J Ambulatory Care Man-agement, 30(3). 